\title{
Higher order super-twisting for perturbed chains of integrators
}

\author{
Salah Laghrouche, Mohamed Harmouche, Yacine Chitour
}

\begin{abstract}
In this paper, we present a generalization of the supertwisting algorithm for perturbed chains of integrators of arbitrary order. This Higher Order Super-Twisting (HOST) controller is homogeneous with respect to a family of dilations and is continuous. It is built as a dynamic controller (with respect to the state variable of the chain of integrators) and the convergence analysis is performed by the use of a homogeneous strict Lyapunov function which is explicitly constructed. The effectiveness of the controller is finally illustrated with simulations for a chain of integrators of order four, first pure then perturbed, where we compare the performances of two HOST controllers.
\end{abstract}

\section{INTRODUCTION}

Many HOSMC algorithms exist in contemporary literature for control of nonlinear systems with bounded uncertainty. These algorithms are robust, they preserve the insensitivity of classical sliding mode, and maintain the performance characteristics of the closed loop system. Levant for example, has presented a method of designing arbitrary order sliding mode controllers for Single Input Single Output (SISO) systems in [1]. Laghrouche et al. [2] have proposed a two part integral sliding mode based control to deal with the finite time stabilization problem and uncertainty rejection problem separately. Dinuzzo et al. have proposed another method in [3], where the problem of HOSMC has been treated as Robust Fuller's problem. Defoort et al. [4] have developed a robust multi-input multi-output HOSMC controller, using a constructive algorithm with weighted homogeneity based finite time stabilization of an integrator chain. Harmouche et al. have presented their homogeneous controller in [5] based on the work of Hong [6]. Sliding mode with homogeneity approach was also used in [7], [8], to demonstrate finite time stabilization of the arbitrary order sliding mode controllers for SISO systems [1]. A Lyapunovbased approach for arbitrary HOSMC controller design was presented in [9], [10]. In this work, it was shown that a class of homogeneous controllers that satisfies certain conditions, could be used to stabilize perturbed integrator chains.

The main drawback of these controllers is that they produce a discontinuous control signal [11], at least at the origin. In order to build continuous controllers and still have finite time convergence, a standard trick consists in using a relative degree extension as advocated for instance in [12], namely to consider the extra equation $\dot{u}=v$, where $u$ is a control and $v$ is

This research was partially supported by the iCODE Institute, research project of the IDEX Paris-Saclay, and by the Hadamard Mathematics LabEx (LMH) through the grant number ANR-11-LABX0056-LMH in the "Programme des Investissements d'Avenir".

S. Laghrouche is with OPERA Laboratory, UTBM, Belfort, France. salah.laghrouche@utbm. fr

M. Harmouche is with Actility, Paris, France. mohamed.harmouchedactility.com

Y. Chitour is with L2S, Universite Paris 11, CNRS 91192 Gif-surYvette, France. yacine.chitour@lss.supelec.fr a virtual HOSM discontinuous control input. This controller should use the output and its the first $r$ derivatives, where $r$ is the sliding mode order. However, to complete that procedure, it is necessary to know the bounds of the uncertainties and their first derivatives and, more restrictively, to suppose that the time integral of $v$ is uniformly bounded for all initial conditions (see Remark 2).

To overcome this problem and to also get finite time convergence, Kamal et al. [11] propose a generalization of the well-known continuous super-twisting algorithm for high order relative degree system with respect to the output (cf. [13]), ensuring finite time convergence of the sliding variable and its $r$ first derivatives to zero, by using a continuous control signal and only information about the sliding variables and its $r-1$ derivatives [14], [15]. The convergence conditions and Lyapunov analysis have been only given up to order three and a higher order controller is just suggested without proof. Other results solving this problem were proposed in [16] and [17]. These algorithms are not homogeneous and thus they can not achieve the $r$-th order of sliding precision with respect to the sliding variable [7].

In the present paper, we provide a homogenous HOST controller for arbitrary order with a complete argument as well as other HOST controllers with the following properties :

- finite time convergence of the sliding variable and its $r$ first derivatives to zero, by using a continuous control signal and only information about the sliding variables and its $r-1$ derivatives;

- it provides finite-time convergence to $r$-order slidingmode set, and therefore provides sliding accuracy of order $r$ with respect to sliding variable.

- it compensates Lipschitz uncertainties/perturbations.

Our analysis is based on the use of a homogeneous strict Lyapunov function for an extended system. The resulting HOST controllers are continuous at the origin and both ensure finitetime stabilization, first for a pure chain of integrators and then for a perturbed one.

To describe our results, recall that a perturbed chain of integrators of length $r$ reads $\dot{z}_{i}=z_{i+1}$ for $1 \leq i \leq r-1$ and $\dot{z}_{r}=\gamma u+\varphi$ where $\gamma$ is a positive measurable signal lower and upper bounded with known positive constants and both $\dot{\gamma}$ and $\dot{\varphi}$ are bounded by known positive constants. Note that we do not assume yet that the additive perturbation $\varphi$ is bounded.

We first provide a HOST controller for a pure chain of integrators (i.e., $\gamma$ is constant and $\varphi$ is equal to zero) based on standard controllers for a pure chain of integrators verifying in addition a geometric condition. The convergence proof using the HOST controller relies on the existence of a homogeneous strict Lyapunov function $W$ associated with an extended 
system. The construction of $W$ is explicit once the standard controllers for the pure chain of integrators are given.

We then prove these HOST controllers can be used for perturbed chains of integrators. In the case $\gamma$ constant (let say equal to one), one must recall that one can stabilize in finite time such a perturbed chain of integrators without using HOST controllers. Indeed, by setting $z_{r+1}:=u+\varphi$ and $v:=\dot{u}$, one gets $\dot{z}_{r+1}=v+\dot{\varphi}$ and a perturbed chain of length $r+1$ is stabilized with the control $v$ and the bounded uncertainty $\dot{\varphi}$. This can be done at the price of increasing the length of the chain of integrators (i.e., the relative degree of the output), which can be a serious drawback in some applications. This technics is referred to as extension of relative degree, cf. [2], [12]. One should therefore see our HOST solution as an alternative to the increase of the length of the chain of integrators. In the case of non constant $\gamma$ and assuming that $0 \leq \gamma_{m} \leq|\gamma| \leq \gamma_{M}$, we provide a solution for arbitrary length under a smallness condition on $\gamma_{M} / \gamma_{m}-1$ and the extra assumption that $\varphi$ is bounded. Notice that we are able to tune the parameters of our controller in the general case of non constant $\gamma$ in terms of the homogeneous Lyapunov function $W$.

The paper is organized as follows. In Section II, we present our results for the general case, first addressing the stabilization by HOST of a pure chain of integrators of arbitrary order and then explaining how to generalize to a perturbed chain by means of homogeneity arguments. We prove two results, one in the case of constant $\gamma$ and the second one for non constant $\gamma$. In Section III-A, we provide explicit examples of standard controllers for pure chains of integrators which do verify the required geometric condition. We finally demonstrate in Section III-B the efficiency of our HOST algorithm for a perturbed chain of integrators of order four.

Notations and definitions. In this paper, we use $\mathbb{R}$ and $|\cdot|$ to denote the set of real numbers and a fixed norm on $\mathbb{R}^{r}$ respectively, where $r$ is a positive integer. For $\lambda>0$, let $D_{\lambda}$ be the $r \times r$ matrix defined by $\operatorname{diag}\left(\lambda^{r}, \cdots, \lambda\right)$. For $m$ positive integer, let $e_{1}, \cdots, e_{m}$ and $J_{m}$ denote the canonical basis of $\mathbb{R}^{m}$ and the $m$-th Jordan block as $\left(J_{m}\right)_{i j}=\delta_{i, j+1}, 1 \leq i, j \leq$ $m$, respectively. If $M$ is a subset of $\mathbb{R}^{r}$, we use $\bar{M}$ to denote its closure. If $x \in \mathbb{R}$, we denote by $[x]$ the integer part of $x$ i.e., the smallest integer not greater than $x$. We define the function sign as the multivalued function defined on $\mathbb{R}$ by $\operatorname{sign}(x)=x /|x|$ for $x \neq 0$ and $\operatorname{sign}(0)=[-1,1]$. Similarly, for every $a \geq 0$ and $x \in \mathbb{R}$, we use $\lfloor x\rceil^{a}$ to denote $|x|^{a} \operatorname{sign}(x)$. Note that $\lfloor\cdot\rceil^{a}$ is a continuous function for $a>0$ and is of class $C^{1}$ with derivative equal to $a|\cdot|^{a-1}$ for $a \geq 1$. If $V: \mathbb{R}^{r} \rightarrow \mathbb{R}^{p}$ is a differentiable mapping, we use $\partial_{j} V$ to denote the partial derivative of $V$ with respect to the $j$-th coordinate $z_{j}$ and more generally $\partial_{\xi} V$ if $V$ depends on a scalar coordinate $\xi$.

We refer to [12], [18] for the definitions of Filippov differential inclusion and Asymptotic and Finite time stability.

Definition 1: (Homogeneity. cf. [12].) Let $r$ be a positive integer. A function $f: \mathbb{R}^{r} \rightarrow \mathbb{R}$ (a vector field $f: \mathbb{R}^{r} \rightarrow \mathbb{R}^{r}$ or a vector-set function $F: \mathbb{R}^{r} \rightrightarrows \mathbb{R}^{r}$ respectively) is said to be homogeneous of degree $q \in \mathbb{R}$ with respect to the family of dilations $\delta_{\varepsilon}(z), \varepsilon>0$, defined by

$$
\delta_{\varepsilon}(z)=\left(z_{1}, \cdots, z_{r}\right) \mapsto\left(\varepsilon^{p_{1}} z_{1}, \cdots, \varepsilon^{p_{r}} z_{r}\right),
$$

where $p_{1} \cdots, p_{r}$ are positive real numbers (the weights), if for every positive $\varepsilon$ and $z \in \mathbb{R}^{r}$, one has

$$
\begin{aligned}
f\left(\delta_{\varepsilon}(z)\right) & =\varepsilon^{q} f(z) \quad\left(f\left(\delta_{\varepsilon}(z)\right)=\varepsilon^{q} \delta_{\varepsilon}(f(z))\right. \text { or } \\
F\left(\delta_{\varepsilon}(z)\right) & \left.=\varepsilon^{q} \delta_{\varepsilon}(F(z)) \text { respectively }\right) .
\end{aligned}
$$

A differential equation $\dot{x}=f(x)$ (A differential inclusion $\dot{x} \in$ $F(x)$ ) is said to be homogeneous of degree $q \in \mathbb{R}$ with respect to the family of dilations $\delta_{\varepsilon}(z)$ if the vector field $f: \mathbb{R}^{r} \rightarrow \mathbb{R}^{r}$ (vector-set function $F: \mathbb{R}^{r} \rightrightarrows \mathbb{R}^{r}$ is).

\section{HIGHER ORDER SUPER-TWISTING CONTINUOUS FEEDBACK FOR A CHAIN OF INTEGRATOR}

The strategy consists first in building an appropriate feedback for a pure chain of integrator and then in tackling the perturbed case by a homogeneity argument.

\section{A. Stabilization of a pure chain of integrator of arbitrarily order}

Let $r$ be a positive integer. The $r$-th order chain of integrator $(C I)_{r}$ is the single-input control system given by

$(C I)_{r} \quad \dot{z}=J_{r} z+u e_{r}, \quad z=\left(z_{1}, \cdots, z_{r}\right)^{T} \in \mathbb{R}^{r}, \quad u \in \mathbb{R}$.

For $\kappa<0$ and $p>0$ with $p+(r+1) \kappa \geq 0$, set $p_{i}:=$ $p+(i-1) \kappa, 1 \leq i \leq r+1$. For $\varepsilon>0$, let $\delta_{\varepsilon}: \mathbb{R}^{r} \rightarrow \mathbb{R}^{r}$ and $\psi_{\varepsilon}: \mathbb{R}^{r+1} \rightarrow \mathbb{R}^{r+1}$ be the family of dilations associated with $\left(p_{1}, \cdots, p_{r}\right)$ and $\left(p_{1}, \cdots, p_{r+1}\right)$ respectively.

The next proposition gathers a standard result on the stabilization in finite time of $(C I)_{r}$ (cf. [6]) as well as a geometric condition on the homogeneous stabilizing feedback, in the spirit of [9], [10].

Proposition 1: [6], [9], [10]

Let $r$ be a positive integer. There exists a feedback law $u_{0}$ : $\mathbb{R}^{r} \rightarrow \mathbb{R}$, homogeneous of degree $p_{r+1}$ with respect to $\left(\delta_{\varepsilon}\right)_{\varepsilon>0}$ such that the closed-loop system $(C I)_{r}$ with $u_{0}$ is finite time stable and the following conditions hold true:

(i) the function $z \mapsto J_{r} z+u_{0}(z) e_{r}$ is homogeneous of degree $\kappa$ with respect to $\left(\delta_{\varepsilon}\right)_{\varepsilon>0}$ and there exists a continuous positive definite function $V_{1}: \mathbb{R}^{r} \rightarrow \mathbb{R}_{+}$, $C^{1}$ except at the origin, homogeneous with respect to $\left(\delta_{\varepsilon}\right)_{\varepsilon>0}$ of degree $2 p_{r+1}$ such that there exists $c>0$ and $\alpha \in(0,1)$ for which the time derivative of $V_{1}$ along non trivial trajectories of $(C I)_{r}$ verifies $\dot{V}_{1} \leq$ $-c V_{1}^{\alpha}$.

(ii) $\quad z \mapsto \partial_{r} V_{1}(z)$ is homogeneous of non positive degree with respect to $\left(\delta_{\varepsilon}\right)_{\varepsilon>0}$ and $z \mapsto \partial_{r} V_{1}(z) u_{0}(z)$ is non negative over $\mathbb{R}^{r}$.

First of all, notice that $\alpha$ must be equal to $1+\kappa /\left(2 p_{r+1}\right) \geq$ $1 / 2$ and $\partial_{r} V_{1}$ is homogeneous with respect to $\left(\delta_{\varepsilon}\right)_{\varepsilon>0}$ of degree $p+(r+1) \kappa \geq 0$. In [6] (and also see below Subsection III-A1), a feedback law $u_{0}: \mathbb{R}^{r} \rightarrow \mathbb{R}$ satisfying Condition $(i)$ is explicitly built and it has been noticed in [9], [10] that the very same feedback satisfies Condition ( $i i)$. The latter is instrumental for building a super-twisting feedback law as shown in the following theorem.

Theorem 1: Consider the homogeneous mapping $u_{0}: \mathbb{R}^{r} \rightarrow$ $\mathbb{R}$ and the continuous positive definite function $V_{1}: \mathbb{R}^{r} \rightarrow \mathbb{R}_{+}$ 
provided by Proposition 1. For every $k_{P} \geq 1$ and $k_{I}>0$, let $u_{S T}(\cdot)$ be the the dynamic state-feedback controller defined by

$$
\begin{gathered}
\dot{\xi}(t)=-k_{I} \partial_{r} V_{1}(z(t)), \xi(0)=0, \\
u_{S T}(t)=k_{P} u_{0}(z(t))+k_{I} \xi(t),
\end{gathered}
$$

and we refer to $u_{S T}$ as the HOST controller. The feedback connection between $(C I)_{r}$ and (1) gives rise to the dynamical system over $\mathbb{R}^{r+1}$ given by

$$
\begin{aligned}
& \dot{z}(t)=J_{r} z(t)+\left(k_{P} u_{0}(z(t))+k_{I} \xi(t)\right) e_{r}, \\
& \dot{\xi}(t)=-k_{I} \partial_{r} V_{1}(z(t)), \quad \xi(0)=0 .
\end{aligned}
$$

There exist $A, d>0$ so that, if $W: \mathbb{R}^{r+1} \rightarrow \mathbb{R}$ is defined by

$$
W(z, \xi)=\left(V_{1}(z)+\xi^{2} / 2\right)^{2-\alpha}-A z_{r} \xi
$$

then $W$ is positive definite, $C^{1}$ except at the origin, homogeneous with respect to $\left(\psi_{\varepsilon}\right)_{\varepsilon>0}$ and the time derivative of $W$ along non trivial trajectories of (2) verifies $\dot{W} \leq$ $-d W^{1 /(2-\alpha)}$. As a consequence, trajectories of (2) converge to zero in finite time, i.e., $u_{S T}$ stabilizes $(C I)_{r}$ in finite time.

\section{Proof of Theorem 1.}

Consider first the positive definite function $V:=V_{1}+\xi^{2} / 2$, which is homogeneous with respect to $\left(\psi_{\varepsilon}\right)_{\varepsilon>0}$ of degree $2 p_{r+1}$. Using Items $(i)$ and $(i i)$, the time derivative of $V$ along trajectories of (2) verifies $\dot{V}=\dot{V}_{1}+\left(k_{P}-1\right) \partial_{r} V_{1} u_{0} \leq-c V_{1}^{\alpha}$.

For $A>0$, the function $W$ defined in (3) is continuous, $C^{1}$ except at the origin, homogeneous with respect to $\left(\psi_{\varepsilon}\right)_{\varepsilon>0}$ of degree $2(2-\alpha) p_{r+1}=p_{r}+p_{r+1}$. Since $V$ is positive definite, $W$ is also positive definite for $A$ small enough. The time derivative of $W$ along trajectories of (2) verifies

$\dot{W} \leq-c(2-\alpha) V^{1-\alpha} V_{1}^{\alpha}-A k_{P} u_{0} \xi+A k_{I} z_{r} \partial_{r} V_{1}-A k_{I} \xi^{2}$

Since $V \geq V_{1}$ and $2-\alpha \geq 1$, one also has from the previous equation that $\dot{W} \leq-c V_{1}+A k_{P}^{2} u_{0}^{2} / k_{I}+$ $A k_{I}\left|z_{r} \partial_{r} V_{1}\right|-A k_{I} \xi^{2} / 2$. The function $z \mapsto-c V_{1}+$ $A k_{P}^{2} u_{0}^{2} / k_{I}+A k_{I}\left|z_{r} \partial_{r} V_{1}\right|$ is homogeneous of degree $2 p_{r+1}$ with respect to $\left(\delta_{\varepsilon}\right)_{\varepsilon>0}$ and, since $V_{1}$ is definite positive, one has for $A$ small enough,

$$
\dot{W} \leq-\left(c V_{1}+A k_{I} \xi^{2}\right) / 2 .
$$

Clearly, the right-hand side of the previous inequality is negative definite and smaller than $-d W^{1 /(2-\alpha)}$ for some positive constant $d$. Therefore, trajectories of (2) converge to zero in finite time and hence $(C I)_{r}$ is stabilized in finite time by the feedback law $u_{S T}$

Remark 1: If $p+(r+1) \kappa=0$, then $\partial_{r} V_{1}$ is of zero homogeneity degree with respect to $\left(\delta_{\varepsilon}\right)_{\varepsilon>0}$ and $\alpha=1 / 2$. In that case, $W$ is homogeneous with respect to $\left(\psi_{\varepsilon}\right)_{\varepsilon>0}$ of degree $3 p_{r+1}$.

Remark 2: In Subsection III-A1, the feedback $u_{0}$ and the Lyapunov function $V_{1}$ are explicitly built (as done in [6]) and the constant $c$ in the differential inequality $\dot{V}_{1} \leq-c V_{1}^{\alpha}$ is explicitly given. The constant $A$ appearing in Theorem 1 can now be chosen as follows. Let $R_{p}$ and $N_{p}$ be the unit spheres in $\mathbb{R}^{r}$ and $\mathbb{R}^{r+1}$ associated to the weights $p_{i}, 1 \leq i \leq r$ and $1 \leq i \leq r+1$ respectively. Set

$$
\begin{aligned}
& V_{m}=\min _{(z, \xi) \in N_{p}} V^{2-\alpha}, \quad Z_{M}=\max _{(z, \xi) \in N_{p}}\left|z_{r} \xi\right|, \\
& Z_{M}^{1}=\max _{z \in R_{p}}\left|z_{r} \partial_{r} V_{1}\right|, \quad Z_{M}^{2}=\max _{z \in R_{p}} u_{0}^{2}, \quad V_{m}^{1}=\min _{z \in R_{p}} V_{1} .
\end{aligned}
$$

Since $V$ is positive definite, $V_{m}>0$. By taking $A$ as

$$
A \leq \min \left(\frac{V_{m}}{2 Z_{M}}, \frac{c k_{I} V_{m}^{1}}{2\left(k_{P}^{2} Z_{M}^{2}+k_{I}^{2} Z_{M}^{1}\right)}, \frac{2 c}{k_{I}}\right),
$$

then the inequalities $V^{2-\alpha} / 2 \leq W \leq 3 V^{2-\alpha} / 2$, (5) and $\dot{W} \leq$ $-A k_{I} V$ are deduced. Then one can take $d=A k / 4$. The next remark will be used in the proof of Theorem 3 .

Remark 3: Note that the constant $k_{P}$ can be replaced by any continuous function $k_{P}:\left[0, \mathbb{R}^{+}\right) \rightarrow\left[k_{m}, k_{M}\right]$ where $k_{m}, k_{M}$ are positive constants with $k_{m} \geq 1$.

We next apply the previous results to get finite-time convergence of the perturbed chain of integrators defined next by

$$
\dot{z}=J_{r} z+(\gamma u+\varphi) e_{r}
$$

where the time-varying functions $\gamma(\cdot)$ and $\varphi(\cdot)$ are globally Lipschitz over $\mathbb{R}_{+}$and verify the following: there exist $\gamma_{m}, \gamma_{M}>0$ and $\bar{\gamma}, \bar{\varphi} \geq 0$ such that, for every $t \geq 0$ it holds

$$
0<\gamma_{m} \leq \gamma(t) \leq \gamma_{M}, \quad|\dot{\gamma}(t)| \leq \bar{\gamma}, \quad|\dot{\varphi}(t)| \leq \bar{\varphi} .
$$

B. Stabilization of a perturbed chain of integrators: case of $\gamma$ constant

In this subsection, we consider the case of $\gamma$ constant and we obtain the following theorem.

Theorem 2: Consider the perturbed chain of integrators defined by (6), where the time-varying function $\gamma(\cdot)$ and $\varphi(\cdot)$ verify $\gamma \equiv \gamma_{m}$ and (7) respectively. Assume that there exists a continuous homogeneous feedback law $u_{0}$ and a Lyapunov function $V_{1}$ verifying the assumptions $(i)$ and $(i i)$ of Theorem 1 with $p+(r+1) \kappa=0$.

Then, for every positive gains $k_{P} \geq 1$ and $k_{I}>0$, there exists $\lambda_{0}>0$ only depending on the gains and $\bar{\varphi}$ such that, for $\lambda \geq \lambda_{0}$, the dynamic state-feedback controller $u_{S T}^{\lambda}(\cdot)$ defined by

$$
\begin{aligned}
\dot{\xi}^{\lambda}(t) & =-\lambda k_{I} \partial_{r} V_{1}\left(D_{\lambda} z(t)\right), \xi^{\lambda}(0)=0, \\
u_{S T}^{\lambda}(t) & =\left(k_{P} u_{0}\left(D_{\lambda} z(t)\right)+k_{I} \xi^{\lambda}(t)\right) / \gamma_{m},
\end{aligned}
$$

stabilizes (6) in finite-time. In particular, $u_{S T}^{\lambda}(\cdot)$ is continuous.

Proof of Theorem 2. Fix now some $k_{P} \geq 1$ and $k_{I}>0$. For every $\lambda>0$, consider the standard time-coordinate change of variable along trajectories of (6) defined by $y(t)=D_{\lambda} z(t / \lambda)$. Under the hypotheses of the theorem, (6) can be rewritten $\dot{y}=$ $J_{r} y+\left(\gamma_{m} u_{\lambda}+\varphi_{\lambda}\right) e_{r}$, where one has set, for $t \geq 0, u_{\lambda}(t):=$ $u(t / \lambda)$ and $\varphi_{\lambda}(t):=\varphi(t / \lambda)$. Note that, for a. e. $t \geq 0,\left|\dot{\varphi}_{\lambda}\right| \leq$ $\bar{\varphi} / \lambda$.

The feedback connection between (6) and (8) can be written as the time-varying system over $\mathbb{R}^{r+1}$ given by

$$
\begin{aligned}
\dot{y}(t) & =J_{r} z(t)+\left(k_{P} u_{0}(y(t))+k_{I} \xi_{\lambda}(t)\right) e_{r}, \\
\dot{\xi}_{\lambda}(t) & =-k_{I} \partial_{r} V_{1}(y(t))+\dot{\varphi}_{\lambda}, \quad \xi_{\lambda}(0)=\varphi_{\lambda}(0),
\end{aligned}
$$


where $\xi_{\lambda}(t)=\xi^{\lambda}(t / \lambda)+\varphi_{\lambda}(t)$.

Clearly, (9) corresponds to the differential inclusion (2) perturbed by the time-varying vector field over $\mathbb{R}^{r+1}$ given by $\left(0, \cdots, 0, \dot{\varphi}_{\lambda}(t)\right)^{T}$ or, equivalently, by the multifunction $(0, \cdots, 0,[-\bar{\varphi} / \lambda, \bar{\varphi} / \lambda])^{T}$ taking values in the subsets of $\mathbb{R}^{r+1}$. Let $W$ be the Lyapunov function defined in (3). Along non trivial trajectories of (9), one gets, for every $t \geq 0$, that

$$
\dot{W} \leq-d W^{2 / 3}+\partial_{\xi} W \dot{\varphi}_{\lambda}(t) \leq-d W^{2 / 3}+\bar{\varphi}\left|\partial_{\xi} W\right| / \lambda .
$$

According to Remark 1, the homogeneity degree of $\left|\partial_{\xi} W\right|$ is equal to $3 p_{r+1}-p_{r+1}=2 p_{r+1}$, i.e., the homogeneity degree of $W^{2 / 3}$. One deduces from Eq. (10) that there exists $\varphi_{*}>0$ such that $\dot{W} \leq-d W^{2 / 3} / 2$, along trajectories of System (9) if $\bar{\varphi} / \lambda \leq \varphi_{*}$, i.e., $\lambda \geq \lambda_{0}=\bar{\varphi} / \varphi_{*}$. One then concludes as previously.

Remark 4: As noticed in Introduction, the contents of Theorem 2 can be derived without relying on HOST controllers but rather on extending the relative degree of the original system, cf. [2], [12]. The latter technics however requires a longer chain of integrators.

Remark 5: Notice that the choice of $\lambda_{0}$ can be made explicit once $u_{0}$ and $V_{1}$ are explicitly given.

Remark 6: We do not know how to extend the above result to cases where $p+(r+1) \kappa>0$. Indeed, for the above homogeneity argument to work, it is necessary that $W^{1 /(2-\alpha)}$ (with $\alpha=1 / 2$ ) has the same degree of homegeneity as $\partial_{\xi} W$ since $\dot{\varphi}$ is simply bounded. On the other hand, $W^{1 /(2-\alpha)}$ has the same degree of homegeneity as $\partial_{\xi} W \dot{\xi}$ and thus $\partial_{r} V_{1}$ must necessarily be of degree zero. This occurs only if $p+(r+1) \kappa=0$.

C. Stabilization of a perturbed chain of integrators: general case

In this subsection, we apply Subsection II-A to get finite-time convergence of (6) where the time-varying functions $\gamma(\cdot)$ and $\varphi(\cdot)$ are measurable over $\mathbb{R}_{+}$, verify (7) and assuming an extra boundedness hypothesis for $\varphi(\cdot)$. Note that this hypothesis can be explicitelty quantified by using $W$

Remark 7: Note that the technics of extension of relative degree can also be applied in this case, cf. [19].

We now want to derive conditions under which the supertwisting feedback defined in Eq. (1) stabilizes System (6) in finite time. We obtain the following theorem in case $\varphi(\cdot)$ is bounded.

Theorem 3: Consider the perturbed chain of integrators defined by (6), where the time-varying function $\gamma(\cdot)$ and $\varphi(\cdot)$ verify (7). Assume that there exists a continuous feedback law $u_{0}$ and a Lyapunov function $V_{1}$ verifying the assumptions of Theorem 1 with $p+(r+1) \kappa=0$. Moreover assume that, in addition to Hypotheses (7), $|\varphi| \leq \varphi_{M}$ for some known non negative constant $\varphi_{M}$ and set $\delta_{\gamma}=\gamma_{M} / \gamma_{m}-1 \geq 0$.

For every $k_{P} \geq 1, k_{I}>0$ and $\lambda>0$, consider the dynamic state-feedback controller $u_{S T}^{\lambda}(\cdot)$ defined by

$$
\begin{gathered}
\dot{\xi}^{\lambda}(t)=-\lambda k_{I} \partial_{r} V_{1}\left(D_{\lambda} z(t)\right), \xi \lambda(0)=0, \\
u_{S T}^{\lambda}(t)=\left(k_{P} u_{0}\left(D_{\lambda} z(t)\right)+k_{I} \xi^{\lambda}(t)\right) / \gamma_{m} .
\end{gathered}
$$

Then there exist $\delta_{0}, \lambda_{0}>0$ so that if $\delta_{\gamma} \leq \delta_{0}$ and $\lambda \geq \lambda_{0}$, then (6) is stabilized in finite-time by the dynamic state-feedback controller $u_{S T}^{\lambda}(\cdot)$ defined in Eq. (11). In particular $u_{S T}^{\lambda}(\cdot)$ is continuous.

Proof of Theorem 3. As in the proof of Theorem 2, fix $k_{P} \geq 1$ and $k_{I}>0$. For $\lambda>0$, consider the time-coordinate transformation $y(t)=D_{\lambda} z(t / \lambda)$. Under the hypotheses of the theorem, (6) can be rewritten $\dot{y}=J_{r} y+\left(\gamma_{\lambda} u_{\lambda}+\varphi_{\lambda}\right) e_{r}$, where one has set, for $t \geq 0 u_{\lambda}(t)=u(t / \lambda), \gamma_{\lambda}(t)=\gamma(t / \lambda)$ and $\varphi_{\lambda}(t)=\varphi(t / \lambda)$. Then, for a. e. $t \geq 0,\left|\dot{\gamma}_{\lambda}\right| \leq \bar{\gamma} / \lambda$ and $\left|\dot{\varphi}_{\lambda}\right| \leq \bar{\varphi} / \lambda$.

For $t \geq 0$, define $\xi_{\lambda}(t)=-k_{I} \int_{0}^{t} \partial_{r} V_{1}(y(s)) d s+$ $\varphi_{\lambda}(t) / \gamma_{\lambda}(t)$. The feedback connection between (6) and (11) can be written as the time-varying system over $\mathbb{R}^{r+1}$ given by

$$
\left\{\begin{aligned}
\dot{y}= & J_{r} y+\left(\frac{\gamma_{\lambda}(t)}{\gamma_{m}} k_{P} u_{0}(y)+k_{I} \xi_{\lambda}(t)\right. \\
+ & \left.k_{I} \frac{\gamma_{\lambda}(t)-\gamma_{m}}{\gamma_{m}} \xi_{\lambda}(t)\right) e_{r} \\
\dot{\xi}_{\lambda}= & -k_{I} \partial_{r} V_{1}(y)+\frac{\dot{\varphi}_{\lambda}(t) \gamma_{\lambda}(t)-\dot{\gamma}_{\lambda}(t) \varphi_{\lambda}(t)}{\gamma_{\lambda}^{2}(t)} \\
& \xi_{\lambda}(0)=\varphi_{\lambda}(0)
\end{aligned}\right.
$$

Notice that, for $t \geq 0, \frac{\left|\gamma_{\lambda}(t)-\gamma_{m}\right|}{\gamma_{m}} \leq \delta_{\gamma}$. Consider now the Lyapunov function $W$ defined in (3). By taking into account Remark 3, one gets along non trivial trajectories of System (12), for a.e. $t \geq 0$, that

$$
\dot{W} \leq-d W^{2 / 3}+\delta_{\gamma}\left|\partial_{r} W \xi_{\lambda}\right|+\frac{\left|\partial_{\xi} W\right|}{\lambda \gamma_{m}^{2}}\left(\bar{\varphi} \gamma_{M}+\bar{\gamma} \varphi_{M}\right) .
$$

Exactly as for $\left|\partial_{\xi} W\right|,\left|\partial_{r} W \xi\right|$ is homogeneous with respect to $\left(\psi_{\varepsilon}\right)_{\varepsilon>0}$ of degree $2 p_{r+1}$. One deduces that there exist $\delta_{0}$ small enough, $\lambda_{1}>0$ and a positive constant $C_{1}>0$ such that, if $\delta_{\gamma} \leq \delta_{0}$ and $\lambda \geq \lambda_{1}$, then one has for a.e. $t \geq 0$ $\dot{W} \leq-d W^{2 / 3} / 2$. along non trivial trajectories of (12) and one concludes as in Theorem 2 .

\section{EXAMPLES AND SIMULATIONS}

A. Feedbacks $u_{0}$ and Lyapunov functions $V_{1}$ verifying the assumptions of Theorems 2 and 3

We next provide examples of controllers $u_{0}$ and Lyapunov functions $V_{1}$ satisfying the conditions of Theorem 2 for $r \geq 2$. We next assume that $p=1$ and thus $\kappa=-1 /(r+1)$.

1) Hong's controller: Such a controller is simply borrowed from [6]. In that reference, the convergence is proved by using a Lyapunov function $V_{0}$ explicitly constructed for that purpose. The latter function does not match the the assumptions of Theorem 2 and we have to modify it to get the required Lyapunov function $V_{1}$.

Let $l_{1}, \cdots, l_{r}$ positive real numbers. We define, for $i=$ $0, \ldots, r+1$, the functions $v_{0} \equiv 0$ and for $1 \leq i \leq r-1$, $v_{i+1}=-l_{i+1}\left\lfloor\left\lfloor z_{i+1}\right\rceil^{\beta_{i}}-\left\lfloor v_{i}\right\rceil^{\beta_{i}}\right\rceil^{\alpha_{i+1} / \beta_{i}}$, where $\beta_{0}=p_{2}$ and $\left(\beta_{i}+1\right) p_{i+1}=\beta_{0}+1$, and $\alpha_{i}=p_{i+1} / p_{i}$, for $i=1, \ldots, r$. One then takes the controller $u_{0}$ to be equal to the continuous function $v_{r}$. Consider the Lyapunov function $V_{0}=\sum_{i=1}^{r} W_{i}$, where the positive real-valued functions $W_{i}$, 
$1 \leq i \leq r$ are given by $W_{i}=\int_{v_{i-1}}^{z_{i}} w_{i}\left(z_{1}, \cdots, z_{i-1}, s\right) d s$ with $w_{i}=\left\lfloor z_{i}\right\rceil^{\beta_{i-1}}-\left\lfloor v_{i-1}\right\rceil^{\beta_{i-1}}, \quad i=1, \cdots, r$. Then, there exists $l, l_{1}, \cdots, l_{r}>0$ such that the time derivative of $V$ along every non trivial trajectory of $\dot{z}=J_{r} z+u_{0} e_{r}$ satisfies $\dot{V}_{0} \leq$ $-l \bar{V}_{0}^{\frac{2+2 \kappa}{2+\kappa}}$. This proves that $u_{0}$ stabilizes $\dot{z}=J_{r} z+u e_{r}$ to the origin in finite-time. Let $\lambda:=2 /(2 r-1)<1$. Note that $1-\lambda=$ $\beta_{r-1} /\left(1+\beta_{r-1}\right)$. Take now $V_{1}=V_{0}^{\lambda} / \lambda$. A simple computation yields $\partial_{r} V_{1}=\left(\left\lfloor z_{r}\right\rceil^{\beta_{r-1}}-\left\lfloor v_{r-1}\right\rceil^{\beta_{r-1}}\right) / V_{0}^{\frac{\beta_{r-1}}{\beta_{r-1}+1}}$.

One then checks that $\partial_{r} V_{1}$ is homogeneous of degree zero with respect to $\delta_{\varepsilon}$, globally bounded, and continuous except at the origin.

2) Modified Hong's Controller: The following controller is a hybrid form between the continuous controller presented by Hong [6] and a terminal sliding mode approach also presented by Hong et al. in [20]. Note that its form is very close to the controller proposed in [21]. Let $\kappa$, the $l_{i}$ 's, $\alpha_{i}$ 's and the $\beta_{i}$ 's as before. The functions $v_{i}$ are defined as above for $1 \leq i \leq r-1$ but we now set $v_{r}=-l_{r} w_{r}$ with $w_{r}:=\left(\left|z_{r}\right|^{\beta_{r-1}}+\left|\bar{v}_{r-1}\right|^{\beta_{r-1}}\right)^{\alpha_{r} / \beta_{r-1}} \operatorname{sign}\left(z_{r}-v_{r-1}\right)$. The controller $u_{0}$ is then taken equal to $v_{r}$ and it stabilizes $\dot{z}=J_{r} z+u e_{r}$ in finite-time. To see that, consider the positive definite function $V_{0}=\sum_{i=1}^{r} W_{i}$ where, for $i=1, \ldots, r$, one has $W_{i}=\int_{v_{i-1}}^{z_{i}} w_{i}\left(z_{1}, \cdots, z_{i-1}, s\right) d s$ with $w_{i}, 1, \cdots, r-1$ defined as in Hong's controller and $w_{r}$ defined just above. One deduces from an argument entirely similar to that of [6] that there exists $l>0$ such that, one has along trajectories of the closed-loop system $\dot{V}_{0} \leq-l V_{0}^{\frac{2+2 \kappa}{2+\kappa}}$. Finite-time convergence to the origin follows immediately. Finally remark that the feedback control law $v_{r}$ is continuous at zero. One can then apply the results given in Section II. The actual Lyapunov function $V_{1}$ is again taken of the form $V_{0}^{\lambda} / \lambda$ with $\lambda=1 /\left(1+\beta_{r-1}\right)$. A simple computation yields $\partial_{r} V_{1}=V_{0}^{\lambda-1} w_{r}$ which is homogeneous of degree zero with respect to $\left(\delta_{\varepsilon}\right)_{\varepsilon>0}$.

\section{B. Simulations}

In this subsection, we verify the effectiveness of our design through simulations. We deal with a chain of integrator of order four and we show the robustness with respect to perturbations. Consider the fourth order integrator system given by $\dot{z}=$ $J_{4} z+(\gamma u+\varphi(t)) e_{4}$. We study in the following subsections two cases: the first one deals with $\varphi \equiv 0$ and $\gamma \equiv 1$, and the second case considers $\varphi \neq 0$ and $\gamma \neq 1$, both for the continuous and discontinuous HOST controllers corresponding to the Hong's controller $u_{0}^{H}$ and to the modified Hong's controller $u_{0}^{M H}$ and respectively. For all subsequent simulations, the control parameters are tuned as follows: $l_{1}=l_{2}=1, l_{3}=4, l_{4}=8$ and $\kappa=-1 / 5$ with initial condition $z_{1}(0)=-5, z_{2}(0)=2$ and $z_{3}(0)=z_{4}(0)=4$.

\section{Simulation of pure integrator chain for $u=u_{0}$}

We start by stabilizing the pure integrator chain, (i.e., with $\varphi \equiv 0$ ) by the controller $u=u_{0}$ where $u_{0}$ represents either the Hong's controller $u_{0}^{H}$ or the modified Hong's controller $u_{0}^{M H}$ given in Sections III-A1 and III-A2 respectively. Figures 1(a) and 1(c) represents $u_{0}^{H}$ and $u_{0}^{M H}$ presented in Sections III-A 1 and III-A 2 respectively. These controllers force the states $\left(z_{1}, z_{2}, z_{3}, z_{4}\right)$ to zero in finite time, as shown in Figure 1(b) and Figure 1(d).

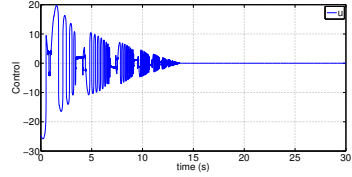

(a) Control law $u$ versus time $(s)$

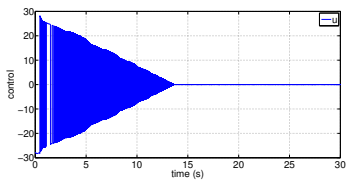

(c) Control law $u$ versus time $(s)$

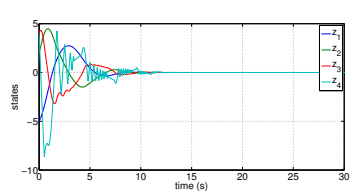

(b) $z_{1}, z_{2}, z_{3}$ and $z_{4}$

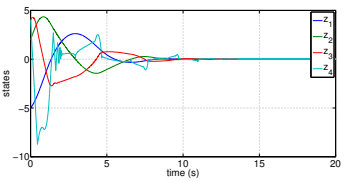

(d) $z_{1}, z_{2}, z_{3}$ and $z_{4}$.
Fig. 1. Pure integrator chain without integration action: (a) and (b) Hong's controller. (c) and (d) Modified Hong's controller

\section{Stabilisation of pure integrator chain by $\operatorname{HOST}-\varphi \equiv 0$}

In this subsection, we show the performance of HOST for the pure integrator chain for $u=k_{P} u_{0}-k_{I} \int \partial_{4} V_{1} d t$. The simulation parameters related to $u_{0}$ with the initial condition are tuned as in the previous subsection. The gains $k_{P}$ and $k_{I}$ are chosen as $k_{P}=k_{I}=1$. The states convergence to zero is presented in Figure 2(b) and 3(b) for the Hong's based controller and the modified Hong's based controller respectively. Figure 2(a) and Figure 3(a) show the control law of Hong's based controller with integral action and the modified Hong's based controller with integral action respectively. Figures 2(c) and 3(c) show the continuous integrator action which vanishes to zero as there is no perturbation to compensate.

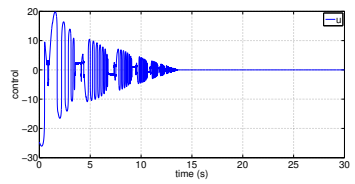

(a) Control law $u$

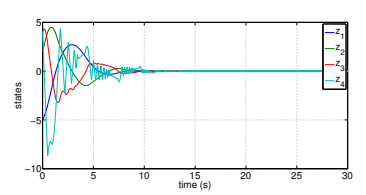

(b) $z_{1}, z_{2}, z_{3}$ and $z_{4}$

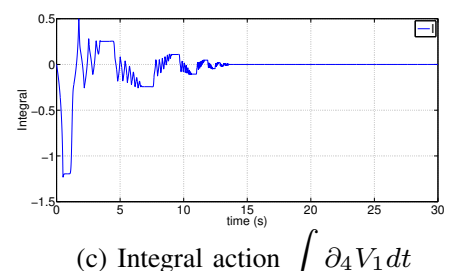

Fig. 2. Pure integrator chain with integral action (with Hong's controller) 


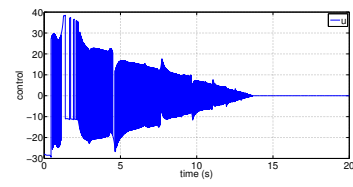

(a) Control law $u$

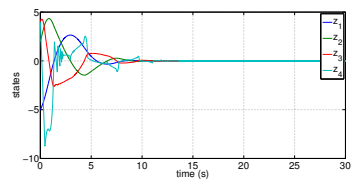

(b) $z_{1}, z_{2}, z_{3}$ and $z_{4}$

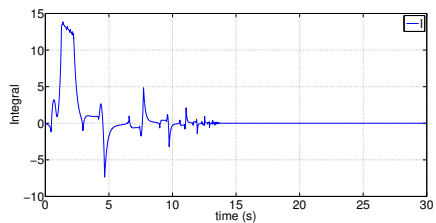

(c) Integral action $I=\int \partial_{4} V_{1} d t$

Fig. 3. Pure integrator chain with integral action (with modified Hong's controller)

E. Stabilisation of perturbed integrator chain by $\mathrm{HOST}-\varphi \neq$ $0, \gamma$ non constant

We now consider the case of a perturbed system with perturbations $\varphi$ and $\gamma$ defined as

$$
\begin{aligned}
\varphi(t) & =\sin (t) \\
\gamma(t) & =3+\frac{1}{2} \sin (0.5 t)
\end{aligned}
$$

Clearly, $\varphi$ is bounded and globally Lipschitz, as well as $\gamma$ which is in addition positive.

The result is similar to the previous cases. However the controller acts in order to compensate the perturbation and we can see clearly in Figure 4(a) and Figure 4(c) for the Hong's based controller that $u(t)=-\frac{\varphi(t)}{\gamma(t)}$ after states convergence to zero. Similar results are obtained in the case of the modified Hong's based controller in Figure 5(a) and Figure 5(c).

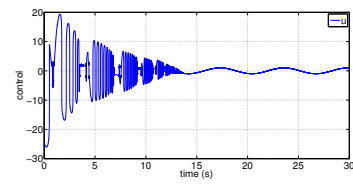

(a) Control law $u$

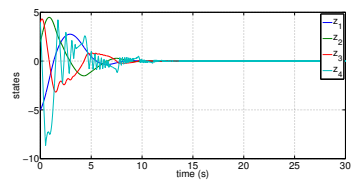

(b) $z_{1}, z_{2}, z_{3}$ and $z_{4}$

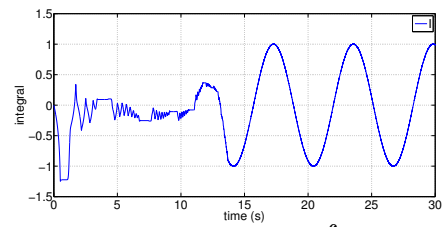

(c) Integral action $I=\int \partial_{4} V_{1} d t$

Fig. 4. Perturbed integrator chain with integral action (with Hong's controller)

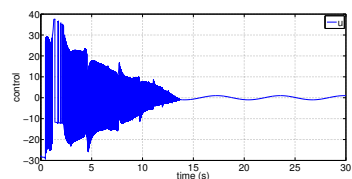

(a) Control law $u$

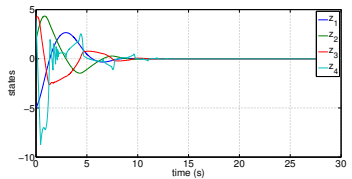

(b) $z_{1}, z_{2}, z_{3}$ and $z_{4}$

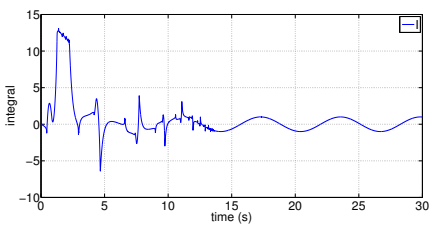

(c) Integral action $I=\int \partial_{4} V_{1} d t$

Fig. 5. Perturbed integrator chain with integral action (Modified Hong's controller)

\section{CONCLUSION}

In this paper we propose a general approach to design a continuous controller for a perturbed chain of integrators of arbitrary order generalizing the well-known supertwisting algorithm provided in [11] for integrator chain of length one and two. We have first designed a controller for the pure chain of integrators using a geometric condition inspired from [9], [10] and we have proved convergence in finite time for the corresponding closed-loop system thanks to the explicit construction of strict homogeneous Lyapunov function for an extended system. As for the perturbed chain of integrators, we partially solve the complete problem by using homogeneity arguments applied to an extended differential inclusion. Future work consists of addressing the general case of a perturbed chain of integrators.

\section{REFERENCES}

[1] A. Levant. Universal single-input-single-output (siso) slidingmode controllers with finite-time convergence. IEEE Transactions on Automatic Control, 46(9):1447-1451, 2001.

[2] S. Laghrouche, F. Plestan, and A. Glumineau. Higher order sliding mode control based on integral sliding mode. Automatica, 43(3):531 - 537, 2007.

[3] F. Dinuzzo and A. Ferrara. Higher order sliding mode controllers with optimal reaching. IEEE Transactions on Automatic Control, 54(9):2126-2136, 2009.

[4] Michael Defoort, Thierry Floquet, Annemarie Kokosy, and Wilfrid Perruquetti. A novel higher order sliding mode control scheme. Systems \& Control Letters, 58(2):102 - 108, 2009.

[5] M. Harmouche, S. Laghrouche, and M. El Bagdouri. Robust homogeneous higher order sliding mode control. In 2011 50th IEEE CDC and ECC, pages 5665-5670, 2011.

[6] Y. Hong. Finite-time stabilization and stabilizability of a class of controllable systems. Systems \& Control Letters, 46(4):231 236, 2002.

[7] A. Levant. Higher-order sliding modes, differentiation and outputfeedback control. International Journal of Control, 76(9-10):924941, 2003.

[8] A. Levant. Homogeneity approach to high-order sliding mode design. Automatica, 41(5):823 - 830, 2005.

[9] M. Harmouche, S. Laghrouche, and Y. Chitour. Robust and adaptive higher order sliding mode controllers. In 51st IEEE Conference on Decision and Control, pages 6436-6441, 2012. 
[10] S. Laghrouche, M. Harmouche, F. S. Ahmed, and Y. Chitour. Control of PEMFC air-feed system using lyapunov-based robust and adaptive higher order sliding mode control. IEEE Transactions on Control Systems Technology, 23(4):1594-1601, 2015.

[11] S. Kamal, A. Chalanga, J. A. Moreno, L. Fridman, and B. Bandyopadhyay. Higher order super-twisting algorithm. In 13th International Workshop on Variable Structure Systems (VSS), pages 1-5, 2014.

[12] Arie Levant. Finite-Time Stability and High Relative Degrees in Sliding-Mode Control, pages 59-92. Springer Berlin Heidelberg, Berlin, Heidelberg, 2012.

[13] A. Levant. Sliding order and sliding accuracy in sliding mode control. International Journal of Control, 58(6):1247-1263, 1993.

[14] S. Kamal, J.A. Moreno, A. Chalanga, B. Bandyopadhyay, and L. Fridman. Continuous terminal sliding-mode controller. Automatica, 69:308 - 314, 2016.

[15] L. Fridman, J.A Moreno, B. Bandyopadhyay, S. Kamal, and A; Chalanga. Continuous Nested Algorithms : The Fifth Generation of Sliding Mode Controllers, pages 5-35. Springer International Publishing, Cham, 2015.

[16] M. Basin, P. Rodriguez-Ramirez, S. Ding, and S. Dominic. A nonhomogeneous super-twisting algorithm for systems of relative degree more than one. Journal of the Franklin Institute, 352(4):1364 - 1377, 2015.

[17] C. Edwards and Y. B. Shtessel. Adaptive continuous higher order sliding mode control. Automatica, 65:183 - 190, 2016.

[18] E. Bernuau, D. Efimov, W. Perruquetti, and A. Polyakov. On an extension of homogeneity notion for differential inclusions. In European Control Conference, pages 2204-2209, 2013.

[19] A. Levant and L. Alelishvili. Integral high-order sliding modes. IEEE Transactions on Automatic Control, 52(7):1278-1282, 2007.

[20] Y. Hong, G. Yang, D. Cheng, and S. Spurgeon. Finite time convergent control using terminal sliding mode. Journal of Control Theory and Applications, 2(1):69-74, 2004.

[21] Y. Hong, G. Yang, D. Cheng, and S. Spurgeon. A new approach to terminal sliding mode control design. Asian Journal of Control, 7(2):177-181, 2005. 